\title{
Analysis of Microstrip Circuit by Using Finite Difference Time Domain (FDTD) Method
}

\author{
ZHANG Lei, YU Tong-bin, QU De-xin and XIE Xiao-gang \\ PLA University of Science and Technology \\ NanJing, China \\ zhanglei_chaoshen@163.com
}

\begin{abstract}
The microstrip circuit is mostly analyzed in transform domain, because its equivalent circuit equation is often nonlinear differential equation, which is easily analyzed in transform domain relatively, but hardly did in time domain, so the analysis of microstrip circuit is a hard work in time domain. In this paper, the FDTD method is used to analyze the microstrip circuit in time domain, by transforming the nonlinear differential equation into time domain iterative equation, selecting suitable time step, and having an iterative computing, the time domain numerical solution can be solved. The FDTD method analyzing the microstrip circuit provides a new way of thought for analyzing microstrip circuit in time domain.
\end{abstract}

\section{Keywords-microstrip; cirtuit; FDTD}

\section{INTRODUCTION}

In the analysis of microstrip circuit, there is often a question that is how to solve the nonlinear differential equation, because the equivalent circuit equation is usually a nonlinear differential equation. For the ordinary differential equation, there has been a relatively complete mathematic method, which can solve the accurate analytical solution, but for the nonlinear differential equation, there is no effective method which can get the accurate analytical solution in time domain. In most case, people want to get the time domain accurate analytical solution for time domain analysis. Some analytical solution can be solved in transform domain, however the transform domain solution is hard to be transformed into the time domain, so the time domain accurate analytical solution is hard to be got.

The FDTD method is an effective method in solving the numerical solution of differential equation, which is widely used to solve the numerical solution of ordinary differential equation, and there are other methods ${ }^{[1-3]}$. The equivalent circuit equation of microstrip circuit is often nonlinear differential equation, we can use the FDTD method to make different discrete transform for the equivalent equation, transform the microstrip circuit into differential equation, have iterative computation, and solve the time domain numerical solution.

In this paper, we take a frequency-halving microstrip circuit as example, and analyze its equivalent circuit equation by FDTD method, solve the time domain numerical solution, which is according with the measured result given in the reference. The result validates that the method is available. It gives a new method to analyze the microstrip circuit.

\section{THEORY ANALYSIS}

The basic thought of FDTD method solving differential equation is making a discrete transformation to the differential equation, and using finite difference instead of the differential coefficient calculation, making time domain iterative calculation, then working out the numerical solution.

\section{A. Difference principle:}

There is an analytic function $\mathrm{y}$, its variable is $\mathrm{x}$, written as $y=f(x)$, the derivative of $\mathrm{y}$ to $\mathrm{x}$ is defined as follow:

$$
\frac{d y}{d x}=\lim _{\Delta x \rightarrow 0} \frac{\Delta y}{\Delta x}=\lim _{\Delta x \rightarrow 0} \frac{f(x+\Delta x)-f(x)}{\Delta x}
$$

Where $d y, d x$ are tiny variable.

So, when $\mathrm{dx}$ is enough tiny, the derivative of $\mathrm{y}$ can be instead of by difference quotient.

There are three common difference forms, they are follows:

Forward difference :

$$
\begin{gathered}
\Delta y=f(x+\Delta x)-f(x) \\
\text { Backward difference : } \\
\Delta y=f(x)-f(x-\Delta x)
\end{gathered}
$$

Center difference :

$$
\Delta y=f\left(x+\frac{1}{2} \Delta x\right)-f\left(x-\frac{1}{2} \Delta x\right)
$$

Formula (1) shows the first order derivative, the difference is called as first order difference. If we made a difference to the first order difference again, we will get the second order difference, written as $\Delta^{2} y$. And so on, we can get every order difference by this method. We take the forward difference as an example.

$$
\begin{aligned}
\Delta^{2} y & =\Delta(\Delta y) \\
& =\Delta[f(x+\Delta x)-f(x)] \\
& =\Delta f(x+\Delta x)-\Delta f(x) \\
& =[f(x+2 \Delta x)-f(x+\Delta x)]-[f(x+\Delta x)-f(x)] \\
& =f(x+2 \Delta x)-2 f(x+\Delta x)+f(x)
\end{aligned}
$$

According to the derivative define, when the difference of variable tends to 0 , the derivative can be calculated by difference quotient. So we can use difference quotient to 
replace derivative in the numerical calculation. We take the center difference form as example, the first order derivative can be written as:

$$
f^{\prime}(x) \approx \frac{f(x+\Delta x)-f(x-\Delta x)}{2 \Delta x}
$$

The second order derivative can be written as:

$$
f^{\prime \prime}(x) \approx \frac{f(x+\Delta x)-2 f(x)+f(x-\Delta x)}{\Delta x^{2}}
$$

B. The finite difference transform of difference equation. There is a difference equation as follow:

$$
\left\{\begin{array}{l}
\frac{d y}{d x}+y^{3}(x)=\sin x \\
y(0)=0
\end{array}\right.
$$

By forward difference transform, it can be written as:

$$
\left\{\begin{array}{l}
y(x+\Delta x)=y(x)-\Delta x *\left(y^{3}(x)-\sin x\right) \\
y(0)=0
\end{array}\right.
$$

Setting $\quad x=n^{*} \Delta x(n=1,2,3 \cdots), \Delta x=10^{-2} \quad, \quad$ by iterative calculation, we can get the numerical solution as follow.
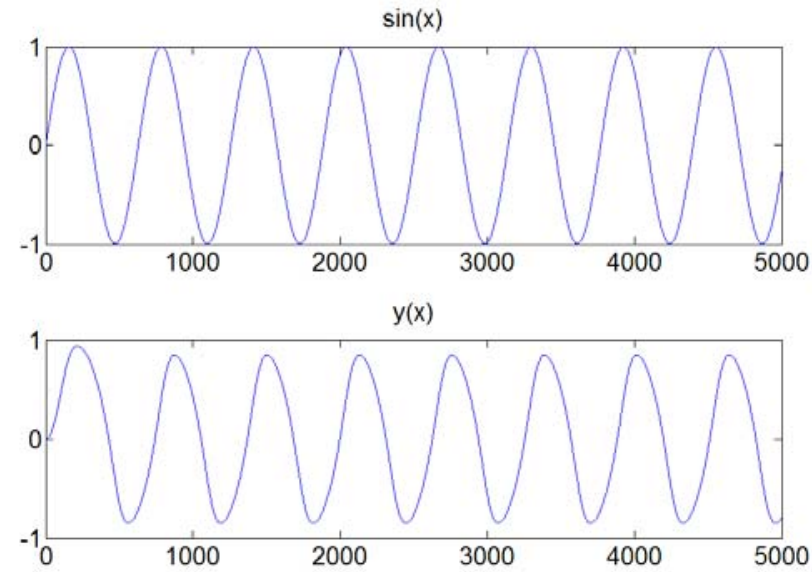

Figure1. The numerical solution of the nonlinear difference equation

Figure1 shows the numerical solution of the equation (8). It can be clearly seen that the numerical solution is stable in the figure. If we take the curve-fitting algorithm, we can get the semi-analytical solution of the equation (8). It is very helpful in the analysis of microstrip circuit, because in the microstrip circuit, the excitation source is often the trigonometric function.

\section{CALCULATION EXAMPLE}

The frequency halver is a special phenomenon in microstrip circuit ${ }^{[4-6]}$, which is very useful in the design of microwave circuit and engineering application. Scholars only found the phenomenon in experiments, but have not analyzed this phenomenon comprehensively in theory. By now there is no breakthrough in principle analysis. Some scholars analyzed the frequency halver microstrip circuit and only solved out the analytical solution of the simplified equivalent circuit equation by complexity mathematical analysis. They got the measure result, but didn't give the numerical analysis in time domain. We analyze the circuit transient response in time domain completely, and take this microstrip circuit ${ }^{[4]}$ as the example of using FDTD method to analyze microstrip circuit, and calculate the time domain numerical solution. The frequency halver microstrip circuit is showed in figure 2.

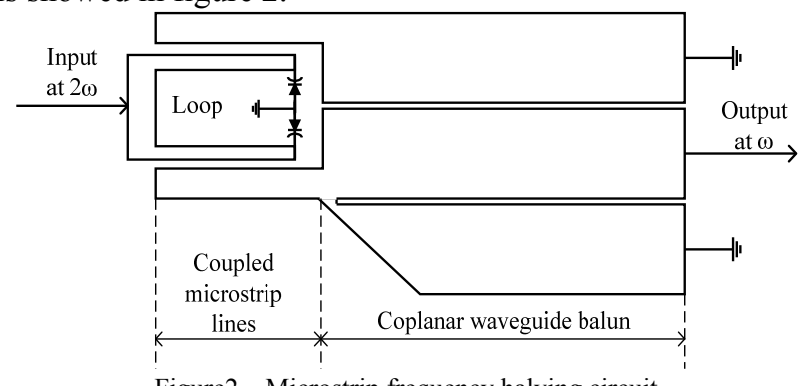

Figure2. Microstrip frequency halving circuit

Figure 2 shows the frequency halving circuit. It can be seen that the frequency halving circuit is symmetrical except the balun. The function of the balun is making the circuit balance. The Loop is a current loop. The nonlinear feature of the varactor can produce various frequency components, which maybe add or subtract through the balanced circuit.

The equivalent circuit is showed in figure 3.

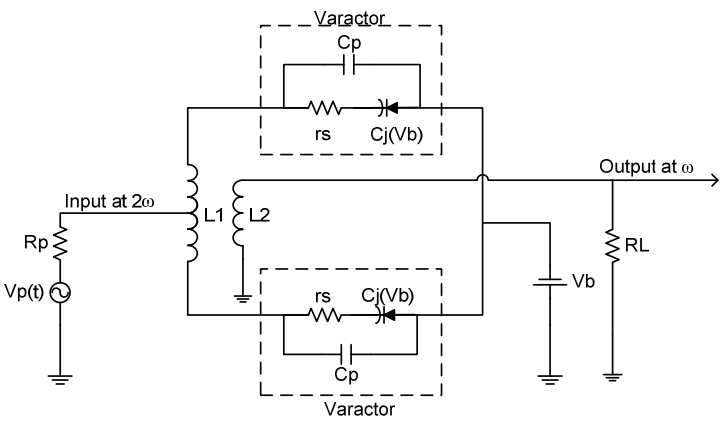

Figure3. The equivalent circuit of frequency halving circuit

Figure 3 shows the equivalent circuit of the frequency halving circuit. It can be seen that the circuit is a symmetrical circuit. Where $V p(t)$ is the alternating current source, $R_{p}$ is the resistance of the $V p(t), R_{L}$ is the load resistance, $\mathrm{L} 1$ and $\mathrm{L} 2$ are the coupling inductance, $\mathrm{Vb}$ is direct current source. The frequency halving phenomenon is produced by the coupling L1 with L2.

The varactors in figure 3 are modeled as

$$
\frac{V(t)}{V_{0}}=\left(1+\frac{q(t)}{Q_{0}}\right)^{2}-1
$$

Where

$$
\begin{aligned}
& V_{0}=\phi_{0}+V_{b} \\
& Q_{0}=2 V C_{j}\left(V_{b}\right)
\end{aligned}
$$

$\phi_{0}$ is the built-in potential, and $V_{b}$ is the reverse bias voltage.

The equation of the equivalent circuit is as follow: 


$$
\begin{aligned}
& (\xi+\xi p) * u^{\prime}+u+\frac{1}{4} *\left(u^{2}+z^{2}\right)=x(t) \\
& z^{\prime \prime}+\xi * z^{\prime}+z+\frac{1}{4} * z=\frac{y^{\prime}}{\xi_{L}} \\
& z^{\prime \prime}=\frac{1}{2} * y+\frac{y^{\prime}}{\xi_{L}}
\end{aligned}
$$

Where, the $\mathrm{u}$ is the sum of varactor charges, $\mathrm{z}$ is the difference between varactors, $x$ is the pumping voltage, $y$ is the output, $\xi_{p}$ is the pumping damping, $\xi_{\mathrm{L}}$ is the load damping, $\xi$ is the ratio of resonance to cutoff frequencies. In the actual circuit, the $\xi_{\mathrm{p}}$ is smaller, and the $\xi_{\mathrm{L}}$ bigger, so the equivalent equation can be simplified as follow:

$$
\begin{aligned}
& \xi * u^{\prime}+u+\frac{1}{4} *\left(u^{2}+z^{2}\right)=x(t) \\
& z^{\prime \prime}+\xi * z^{\prime}+z+\frac{1}{4} * z=0 \\
& z^{\prime \prime}=\frac{1}{2} * y
\end{aligned}
$$

Making a finite different transformation for the equation (13), it can be written as follow:

$$
\begin{aligned}
& u^{n+1}=u^{n-1}-\frac{2 \Delta t}{\xi} *\left\{\frac{1}{4}\left[\left(u^{n}\right)^{2}+\left(z^{n}\right)^{2}\right]-x^{n}+u^{n}\right\} \\
& z^{n+1}=z^{n-1}-\frac{2 \Delta t}{\xi} *\left(z^{n}+\frac{1}{4} u^{n} z^{n}+\frac{1}{2} y^{n}\right) \\
& y^{n}=\frac{2}{(\Delta t)^{2}} *\left(z^{n+1}-2 z^{n}+z^{n-1}\right)
\end{aligned}
$$

Where the $\mathrm{n}$ is the number of time step, $\Delta t$ is time step. The numerical solution can be solved by iterative calculation equation (14), the result are showed in figure 4. The initial condition is $\xi=0.1, \mathrm{u}(0)=0.5, \mathrm{z}(0)=0, \mathrm{z}^{\prime}(0)=0.2$, $x(t)=\cos (2 t)$. In the iterative calculation, if the equation(12) don't be simplified, and the $\xi_{\mathrm{L}}$ is took into the calculation, the iterative calculation will be unstable. The $\xi_{\mathrm{L}}$ is bigger so that it makes a violent change into the iterative calculation.

Figure 4(a), (b) show the transient solutions, figure4(c), (d) show the frequency domain transforming solutions. It can be clearly seen that the output frequency is the half of the input frequency from figure 4 (c), (d). It indicates that the microstrip circuit shown in figurel has the frequencyhalvering function. The numerical solution agrees with the measured result given in the reference [4]. It proves that the method is viable in analysis microstrip circuit.
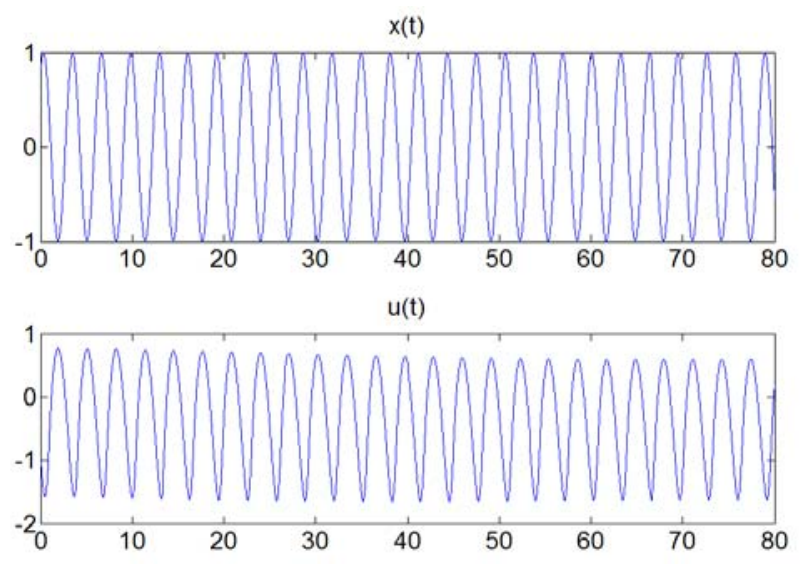

(a)

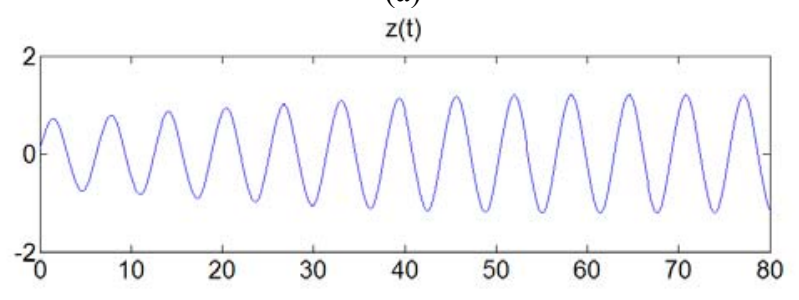

$y(t)$

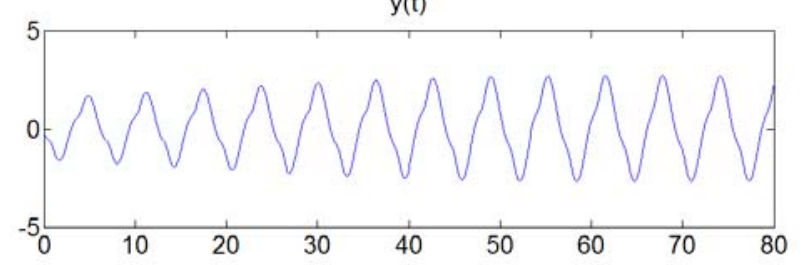

(b)

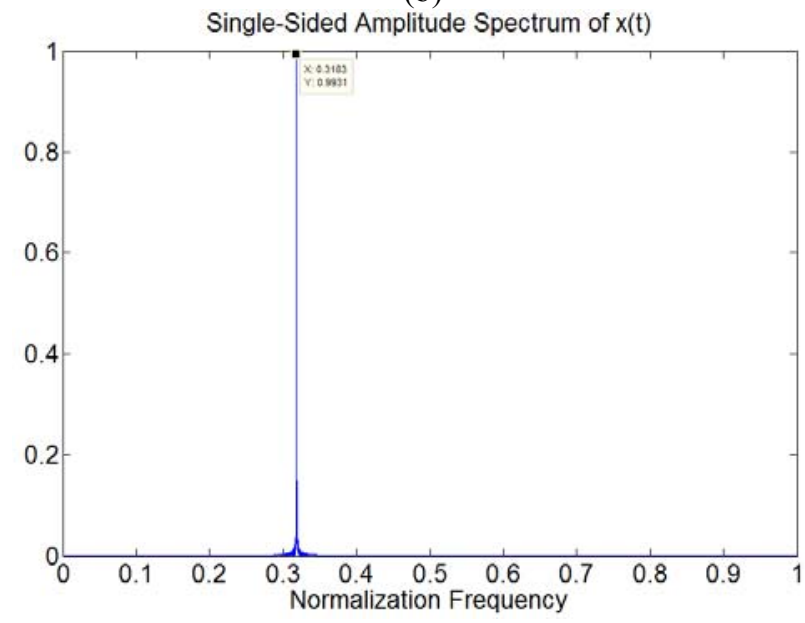

(c) 


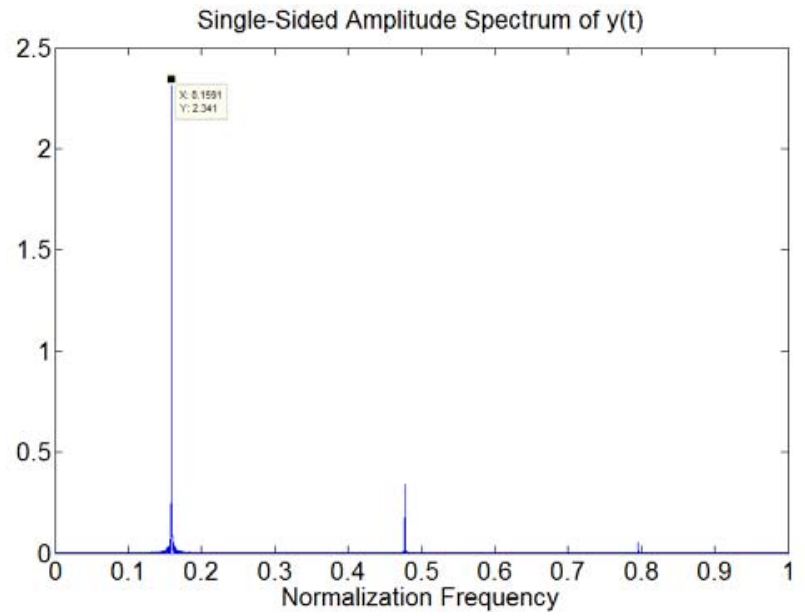

(d)

Figure4. The numerical solution by FDTD method

When using the method to analyze the microstrip circuit, there are two key points. The first is that setting suitable time step $(\Delta t)$ makes the simulation stable, and the second is that the timing sequence of responses must be according with the actual circuit. In study, we try set $x(t)$ as different signals such as direct spread spectrum signals, FM signals, and double frequency signals, all the numerical solutions can be solved, and show the frequency-halving phenomenon. It provides that the method is effective and the circuit is very helpful in engineering application.

\section{CONCLUSION}

In this paper, we use the FDTD method to analyze the frequency-halving microstrip circuit, and solve out the numerical solution by iterative calculation. The numerical solution is according with the measured results. It shows that the method is feasible, and it provides a new thought for the time domain analysis and engineering design of microstrip circuit.

\section{REFERENCES}

[1] LIN Shuang, ZHANG Jie. Analysis of Basic Numerical Solutions for the Initial Value Problem of Ordinary Differential Equations [J]. Journal of Hainan Normal University: Natural Science ,2012,25(2):119-121.

[2] Wang JianQiang,Shen YuLe. Numerical Calculation Analysis on Different Methods of Differential Equation[J]. URBAN GEOTECHNICAL INVESTIGATION \& SURVEYING, $2010,(4): 117-119$

[3] SHANG Zhi, LIU Rui-lan, SU Guang-hui et al. Numerical Method of Differential Equation with Neural Networks[J]. CHINESE JOURNAL OF COMPUTATIONAL PHYSICS, 2001,18(3):281-284

[4] Robert G, Harrison. Theory of the Varactor Frequency Halver[J]. IEEE MTT-S Digest, 1983, 203-205

[5] Robert G, Harrison. A Broad-Band Frequency Divider Using Microwave Varactors[J]. IEEE TRANSACTIONS ON MICROWAVE THEORY AND TECHNIQUES, 1977, NO 12:10551059

[6] Grigorios A, kalivas, Robert G, Harrison. A new Slotline-Microstrip Frequency Halver[J].IEEE MTT-S Digest.1985,683-686. 